evidence from East Africa permit similar conclusions, including pollen analyses, geomorphological studies, lake-level changes and the pattern of sedimentation in the eastern Mediterranean Sea. What is of much greater importance is the demonstration that this technique is capable of documenting past animal diets, and hence environments, with such apparent reliability. It offers, therefore, a valuable means of investigating environmental changes in any dry areas where bone or tooth material is available.

Peter D. Moore is in the Division of Biosphere Sciences, King's College, Campden Hill Road, London W8 $7 A H$, UK.

\title{
Making light work of logic
}

\section{Robert W. Keyes}

INTEREST in the dissipation of energy in the logical operations in a computer dates from the early days of transistorized computation, when it was recognized that solid-state devices promised substantial reductions in energy use through miniaturization ${ }^{1}$. Thinking about the subject was revolutionized when Charles Bennett explained that dissipation could be reduced to an arbitrarily low level in principle by arranging to do computation in a thermodynamically reversible way ${ }^{2}$. The logic functions commonly used - for example, an AND function with two inputs and one output - are inherently irreversible, however: the output does not contain enough information to allow the physical operations to be reversed and the inputs to be reconstructed. The 'Fredkin gate' avoids this logical irreversibility". Now G. J. Milburn of the University of Queensland suggests that a Fredkin gate might be implemented with photons, even with single-photon signals ${ }^{4}$.

The Fredkin gate receives three input signals $a, b, c$, and creates three corresponding outputs, $A, B, C$. Its logical functioning is shown in the table and can be simply described ${ }^{3}$. Input $c$ passes through the gate unchanged to become output $C$. The value of $c$ determines what happens to $a$ and $b$ : if $c$ is zero, all bits are transmitted unchanged; if bit $c$ is one, $a$ and $b$ are interchanged, $a$ emerges as $B$ and $b$ as $A$. It is clear that the inputs can be reconstructed from the outputs.

Milburn proposes to operate a MachZehnder interferometer as a Fredkin gate. The Mach-Zehnder interferometer is designed to reveal the difference in optical path length between two beams that traverse different arms of the instrument (see figure). In the optical gate each arm contains a nonlinear optical material, a material whose index of refraction depends on the intensity of the light within it. The signal beams representing $a$ and $b$ enter the interferometer and are combined so that the intensity in each arm is the same.

The nonlinear element in one of the arms can be irradiated with a beam of light that does not pass through the interferometer and represents input $c$. If there is no external radiation applied as $c$, the beams emerging from the arms interfere to reproduce the original inputs in separate beams. However, application of light of the correct intensity to this controlled arm changes its index of refraction. The phase of the interferometer beam passing through it is changed, so when the beams interfere at the output the location of the emerging beams is reversed. It is also assumed that the control signal passes through the device unaltered to produce output $C$. If each beam contains only one photon,

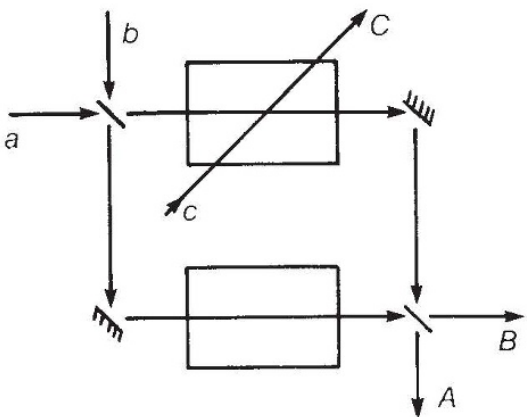

Mach-Zehnder interferometer, as modified by Milburn ${ }^{4}$ for reversible logic operations. Input $(a, b)$ and output $(A, B)$ signals are split and mixed by half-silvered mirrors; by changing the refractive index of nonlinear optical material, signal $c$ alters the path length along one arm of the interferometer.

one has a single-photon Fredkin gate.

Logical reversibility is no guarantee of dissipationless logic; the devices used to build the gate must themselves be free of energy dissipation. In this case, the photons emerging from the single photon gate are presumably identical to those entering, so no energy is lost in the components, and the operation is dissipationless. The use of photons effectively insulates the gate from thermal noise. Photons in the optical region of the spectrum have an energy at least 100 times the thermal energy $k T$, so that they are unlikely to be produced by a thermal fluctuation.

Milburn is quite frank about the practicality of the proposal. He points out that optical nonlinearities are notoriously weak and that a strong electric field cannot be expected from a one-photon signal. Careful adjustment and very accurate beam-splitting is needed to achieve just the right phase relations. Error-free operation demands that the system be lossless.

One may also wonder what is necessary

\begin{tabular}{cccccc}
\hline \multicolumn{5}{c}{ Logic table for a Fredkin gate } \\
\hline \multicolumn{3}{c}{ INPUT } & & \multicolumn{3}{c}{ OUTPUT } \\
\hline$c$ & $a$ & $b$ & $C$ & $A$ & $B$ \\
\hline 0 & 0 & 0 & 0 & 0 & 0 \\
0 & 1 & 0 & 0 & 1 & 0 \\
0 & 0 & 1 & 0 & 0 & 1 \\
0 & 1 & 1 & 0 & 1 & 1 \\
1 & 0 & 0 & 1 & 0 & 0 \\
1 & 1 & 0 & 1 & 0 & 1 \\
1 & 0 & 1 & 1 & 1 & 0 \\
1 & 1 & 1 & 1 & 1 & 1 \\
\hline
\end{tabular}

to ensure that all of the photons are in the right place at the right time. Comparison with the so-called single-electron transistor is instructive ${ }^{5}$. In this device, which there is no room to describe here, an electron can sit on a capacitor until it is needed to influence a current. There is no analogue to a capacitor in optics.

What of strong signals containing many photons? More work remains to be done with realistic many-photon pulses. Any fluctuations in the number of photons in the beam would affect the phase shift in the nonlinear optical materials and degrade the operation of the gate. Noise or degradation cannot be tolerated in true dissipationless computation. The point is not to perform a single operation and show that it worked, but to carry through a long series of operations, record the result, and then recover stored energy by reversing the computation'? If the sequence is long enough, dissipative end effects associated with recording the result contribute little to the energy cost per operation. So I do not agree with Milburn that no more is required than that "the change in intensity is resolvable above the noise level". In fact, the output of one logic element becomes the input to another. Inputting the noise to the next stage allows the noise to cumulate and grow and produces quick degradation of distinctions between zeros and ones. Currently used dissipative systems control noise with nonlinear response, degrading weak signals (noise) and enhancing strong ones.

In spite of the limitations of the optical Fredkin gate, carefully explained by Milburn, it is fodder for journalists who seek to exaggerate illusions of technological revolutions. According to one report", for example, the development "could have long-term implications for the computer industry" and "might one day enable computers to be built which are enormously more efficient than today's". Milburn himself states that "the model of this paper is offered as a reasonably simple way to explore in more detail possible general quantum limits to computation". $\square$ Robert $W$. Keyes is in the IBM Research Division. Yorktown Heights, New York 10598. USA.

1. Landauer. R. IBM J. Res. Dev. 5. 183-191 (1961). Bennett. C. H. IBM J. Res. Dev. 17. 525-532 (1973)

Fredkin. E. \& Toffoli. T. Int J. theor. Phys. 21. 219 (1982)

Milburn. G. J. Phys. Rev. Lett. 62. 2124-2127 (1989).

Fulton. T. A. \& Dolan. G. J. Phys. Rev. Lett. 59. 109 (1987) 6. New Scientist 122. 32 (10 june 1989). 